\title{
The Early Stages of Extragalactic Star Cluster Evolution: New Results From Gemini
}

\author{
Kelsey E. Johnson \\ JILA, University of Colorado, Boulder, CO, 80309, USA \\ William D. Vacca \\ Max-Planck-Institut fuer extraterrestrische Physik, Postfach 1312, \\ D-85741, Garching, Germany
}

Peter S. Conti

JILA, University of Colorado, Boulder, CO, 80309, USA

Henry A. Kobulnicky

University of Wisconsin, $475 \mathrm{~N}$ Charter St., Madison, WI, 53706, USA

\begin{abstract}
As part of an ongoing program to better understand the early stages of massive star cluster evolution and the physical conditions required for their formation, we have obtained $10 \mu \mathrm{m}$ (N-band) images with Gemini North of the nuclear region of the starburst galaxy He 210. Five massive star clusters still enshrouded in their natal cocoons with no optical counterparts were previously discovered by Kobulnicky \& Johnson (1999) as optically thick thermal radio sources. Three of these five radio sources have strong $10 \mu \mathrm{m}$ detections in only 10 minutes of integration time with Gemini. The blackbody temperatures of the dust cocoons are estimated to range from $\sim 40-150 \mathrm{~K}$ at their outer and inner edges, and the mass of these dust shells is $\sim 10^{7} \mathrm{M}_{\odot}$. The ages of the embedded stellar clusters must be $<10^{6}$ years. The bolometric luminosities of the exciting clusters can be constrained to $\sim 10^{8-9} \mathrm{~L}_{\odot}$, and the implied masses are $>10^{6} \mathrm{M}_{\odot}$. These three embedded clusters are responsible for at least $60 \%$ of the IRAS flux of the entire galaxy He 2-10.
\end{abstract}

\section{Introduction}

Newly born massive star clusters, still embedded in their birth material, have recently been detected in the radio images of a handful of galaxies (e.g. NGC 5253: Turner, Beck, \& Ho 1998; He 2-10: Kobulnicky \& Johnson 1999, NGC 2146: Tarchi et al. 2000). These heavily enshrouded clusters manifest themselves as optically thick free-free radio sources. While such dense, inverted-spectrum HII regions exist around individual stars in the Galaxy (i.e. ultracompact HII regions, Wood \& Churchwell 1989), this phenomenon had not previously been seen on such large mass scales. Because of their spectral similarity to galactic 
ultracompact HII regions, Kobulnicky \& Johnson (1999) dubbed these embedded massive star clusters "ultradense HII regions" (UDHIIs). The properties of these UDHIIs are remarkable; the estimated sizes (a few parsecs), masses $\left(\sim 10^{5-6} \mathrm{M}_{\odot}\right)$, and ionizing luminosities $\left(\mathrm{N}_{L y c} \sim 10^{51-53}\right)$ of the newly discovered objects imply that we may be witnessing the birth process of globular clusters. Studying the genesis of local massive star clusters may yield information about the environment in which globular clusters were formed.

\section{Modeled Properties}

Using the analysis of ultracompact HII regions as a guide (see e.g. Churchwell, Wolfire, \& Wood 1990), we have constructed simplified physical models of the UDHIIs. The $10 \mu \mathrm{m}$ luminosities of the three UDHIIs, in addition to the previously known radio luminosities and estimated IRAS luminosities, allow us to estimate the temperatures, masses, and sizes of the dust cocoons as well as the bolometric luminosities, ages, and masses of the embedded clusters. These models suggest that the dust cocoons are typically $\sim 15 \mathrm{pc}$ in radius, and the dust shells have masses $\sim 10^{7} \mathrm{M}_{\odot}$. The temperatures in the dust shells vary between $\sim 150 \mathrm{~K}$ and $\sim 40 \mathrm{~K}$ at the inner and outer edges. The embedded clusters have masses $>10^{6} \mathrm{M}_{\odot}$ and ages $<10^{6}$ years.

\section{Discussion}

The N-band flux of the three detected sources suggests that at least $60 \%$ of the IRAS flux of the entire galaxy can be attributed to only these three UDHIIs. This result has possible implications for the well known infrared-radio correlation (often parameterized by the logarithmic far-infrared to radio ratio, $q$ ). Over a range of spiral, irregular, and starburst galaxies (see Sanders \& Mirabel 1996), $q$ is very robust with a small standard deviation; $\langle q\rangle=2.35 \pm 0.2$. However, Condon et al. (1991) find $q$ tends to be higher for the more infrared luminous galaxies. For He 2-10 we find $q=2.63$, significantly higher than the typical value derived for starburst galaxies, but consistent with the typical value for ultraluminous infrared galaxies. It is therefore plausible that high $q$ values may be used to identify galaxies which host UDHIIs. Furthermore, because high $q$ values are typical of ultraluminous infrared galaxies, UDHIIs may have a role in providing the infrared luminosities of these galaxies.

\section{References}

Condon, J.J. Huang, Z.-P., Yin, Q.F., \& Thuan, T.X. 1991, ApJ, 37865

Churchwell, E. Wolfire, M.G., \& Wood, D.O.S. 1990, ApJ, 354, 247

Kobulnicky, H.A. \& Johnson, K.E. 1999, ApJ, 527, 154

Sanders, D.B. \& Mirabel, I.F. 1996, ARA\&A, 34, 749

Tarchi, A., et al., 2000, A\&A, 358,95

Turner, J.L., Beck, S.C., \& Ho, P.T.P. 1998, ApJ, 532, L109

Wood, D.O. \& Churchwell, E. 1989, ApJS, 69, 831 


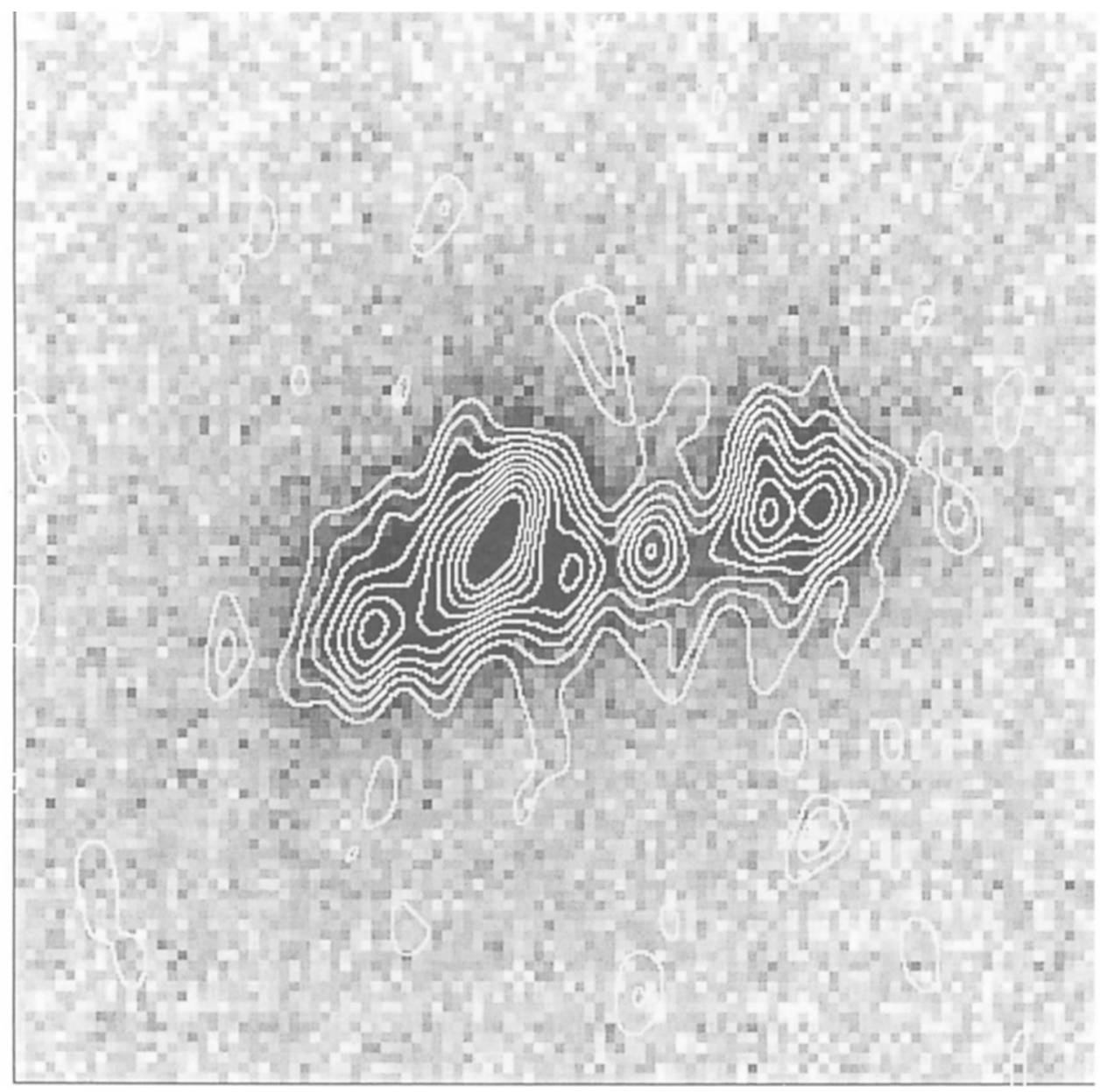

Figure 1. N-band Gemini image of He 2-10 overlaid with $2 \mathrm{~cm}$ radio contours from Kobulnicky \& Johnson (1999). While the the UDHIIs detected at radio wavelengths have no optical counterparts, three of them correspond to bright $10 \mu \mathrm{m}$ sources. 